\title{
Agenesia pulmonar direita na unidade de terapia intensiva adulto
}

\section{Right lung agenesis in the adult intensive care unit}

\author{
Fernando Augusto Pinheiro ${ }^{1,2}$ (D), Laurindo Pereira de Souza ${ }^{3,4}$ (D), Ellen Daiane Biavatti de Oliveira Algeri ${ }^{5}$ (D)
}

1. Docente do departamento de enfermagem pela Faculdade de Pimenta Bueno (FAP), Pimenta Bueno, RO, Brasil. 2. Setor de Terapia Intensiva Adulto do Hospital Regional de Cacoal (HRC), Cacoal, RO, Brasil. 3. Doutorando em Ciências da Saúde pelo Instituto de Assistência Médica ao Servidor Público Estadual de São Paulo (IAMSPE/SP), São Paulo, SP, Brasil. 4. Coordenador da Residência multiprofissional em Cuidados intensivos do Hospital Regional de Cacoal (HRC), Cacoal, RO, Brasil. 5. Doutoranda em Enfermagem pela Universidade de Rio Grande (FURG); Mestre em Gestão e Inovação em saúde pela Universidade Federal do Rio Grande do Norte (UFRN). Enfermeira no Hospital Universitário Dr. Miguel Riet Correa Jr. (HUFURG/EBSERH), Rio Grande /RS, Brasil

\section{Resumo}

Relato de caso: as malformações congênitas pulmonares são raras e variam quanto à sua forma de apresentação clínica e gravidade, podendo manifestarse em qualquer idade. A anamnese e o exame físico foram instrumentos essenciais, além, da entrevista com os familiares para que o diagnóstico de agenesia pulmonar e dextrocardia fossem confirmados de forma rápida e as intervenções de enfermagem e médicas implementadas em tempo recorde e o paciente recebesse alta sem sequelas além das consequências já deixadas pela doença desde sua infância. Conclusão: compreender o diagnóstico é um processo permeado por inquietudes e requer uma avaliação minuciosa dos pacientes a fim de minimizar danos irreversíveis. Sendo assim, a sistematização da assistência de enfermagem é um recurso ímpar que viabiliza a organização da assistência pelo enfermeiro e possibilita utilizar sistemas de classificação da prática de enfermagem, planejamento e avaliação da assistência além de detectar indicadores clínicos, gerando melhora no prognóstico do paciente.

Palavras-chave: Agenesia Pulmonar. Diagnóstico. Sistematização da Assistência de Enfermagem.

\begin{abstract}
Case report: congenital pulmonary malformations are rare and vary in their clinical presentation and severity and may manifest at any age. Anamnesis and physical examination were essential tools, as well as interviews with family members so that the diagnosis of pulmonary agenesis and dextrocardia could be confirmed quickly and nursing and medical interventions implemented in record time and the patient was discharged without sequelae beyond the consequences already left by the disease since its childhood. Conclusion: understanding the diagnosis is a process permeated by concerns and requires a thorough evaluation of patients in order to minimize irreversible damage. Thus, the systematization of nursing care is a unique resource that enables the organization of care by the nurse and enables the use of nursing practice classification systems, planning and assessment of care, as well as detecting clinical indicators that improve patient's prognosis.
\end{abstract}

Key words: Pulmonary Agenesis. Diagnosis. Systematization of nursing care.

\section{INTRODUÇÃO}

As malformações congênitas dos pulmões são raras e variam muito quanto à sua forma de apresentação clínica e gravidade, dependendo, principalmente, do grau de envolvimento pulmonar e de sua localização na cavidade torácica; além disso, elas podem manifestar-se em qualquer idade. Os indivíduos com malformações congênitas dos pulmões podem apresentar sintomas respiratórios ao nascimento, enquanto outros podem permanecer assintomáticos por longos períodos ${ }^{1}$.

A Agenesia Pulmonar (AP) é uma anomalia rara quando comparada com as demais, representando aproximadamente 7,5 a $18,75 \%$ de todas as malformações congênitas. Essa anomalia é, normalmente, assintomática; no entanto, em pacientes sintomáticos, as manifestações clínicas apresentamse inespecíficas, de modo que não há predileção por sexo, e os pulmões são, igualmente, comprometidos, apenas com exceção da agenesia pulmonar esquerda, que é sempre isolada ${ }^{2,3}$.
A agenesia pulmonar é uma má-formação congênita cuja etiologia não é completamente definida, e sua prevalência é de 0,5-1,0 para cada 10.000 nascidos vivos, incluindo tanto a forma bilateral, quanto a unilateral; sendo a forma bilateral incompatível com a vida ${ }^{4}$.

$\mathrm{Na}$ agenesia pulmonar unilateral, o brônquio, fonte de um dos lados, não se desenvolve, o que acarreta a ausência de brônquio, parênquima e vasos pulmonares. Sua taxa de mortalidade no período neonatal é de, aproximadamente, $50 \%$, principalmente, se houver outras malformações associadas, sobretudo as cardíacas ${ }^{4}$.

É importante frisar que a taxa de mortalidade é maior quando a agenesia é no pulmão direito, uma vez que há um maior desvio mediastinal, levando à compressão traqueal.5 No entanto, o acometimento da agenesia é mais prevalente à esquerda, 
com consequente crescimento compensatório do pulmão remanescente e sua herniação no tórax contralateral ${ }^{6}$.

As malformações clínicas são altamente variáveis, podendo ser diagnosticadas na primeira infância, devido às disfunções cardiopulmonares severas ou serem, ocasionalmente, encontradas em pacientes assintomáticos ${ }^{7}$.

Tendo em vista que a agenesia pulmonar é uma anomalia rara, evidencia-se a importância da pesquisa nesta área a fim de ampliar o conhecimento dos profissionais e viabilizar o estabelecimento de condutas específicas com melhora dos desfechos.

Desse modo, é notável que a literatura, voltada para a enfermagem com ênfase na AP, é incipiente; assim, é de fundamental importância o estabelecimento de diagnósticos de enfermagem inerentes ao caso clínico do paciente para permitir agilidade nas escolhas das intervenções e consequente melhoria da qualidade da assistência.

Destarte, o objetivo deste estudo foi apresentar o caso de um paciente admitido na unidade de terapia intensiva adulto com agenesia pulmonar direita as evidências científicas acerca da temática e suas implicações para enfermagem com o intuito de estabelecer o empoderamento necessário ao profissional enfermeiro no exercício de sua prática profissional

\section{RELATO DE CASO}

Paciente do sexo masculino, 21 anos, estudante universitário, procurou o Pronto Socorro Estadual de Cacoal, referência para o Cone Sul de Rondônia, com quadro de dispneia súbita e precordialgia em hemitórax anterior esquerdo, que melhorava com repouso, mantendo nível de consciência na Escala Coma Glagow-15. Foi encaminhado para realização de radiografia de tórax e apresentou piora clínica durante a realização do exame, retornando para a sala vermelha em parada cardiorrespiratória (PCR) por hipóxia não monitorada. Foi realizado um ciclo de reanimação cardiopulmonar cerebral (RCPC), e, em seguida, intubação orotraqueal (IOT), acoplando-o ao ventilador mecânico (VM). Após estabilização do quadro clínico, foi realizada tomografia de tórax, na qual evidenciou-se pneumotórax espontâneo bilateral, sendo realizado toracocentese bilateralmente. Após 24 horas de admissão no pronto-socorro, foi encaminhado para Unidade de Terapia Intensiva Adulto (UTI) do Hospital Regional de Cacoal/ RO, pertencente ao complexo hospitalar estadual-COHREC. Mantinha-se intubado/VM, com sedadoanelgesia (Midazolam e Fentanil em dripping) e droga vasoativa (DVA) (Noradrenalina $0,06 \mathrm{mcg} / \mathrm{Kg} / \mathrm{min}$ ) em dripping; Pressão Arterial (PA) de 74/54 $\mathrm{mmHg}$ e Frequência Cardíaca (FC) de 46 bpm, com resposta efetiva a uma ampola de atropina $0,50 \mathrm{mg} / \mathrm{ml}$. Em seguida, apresentou melhora da perfusão tecidual, saturação de oxigênio de 99\%, mantendo dreno de tórax bilateral; hemitórax esquerdo oscilante com débito hemático e hemitórax direito sem débito e não oscilante.
Figura 1. Radiografia de tórax em incidência anteroposterior, evidenciando pulmão esquerdo com hiperinsuflação compensatória por ausência de pulmão à direita e desvio das estruturas mediastinais à direita, apresentando dextrocardia e dreno de tórax bilateral. (Laudo Radiologista).

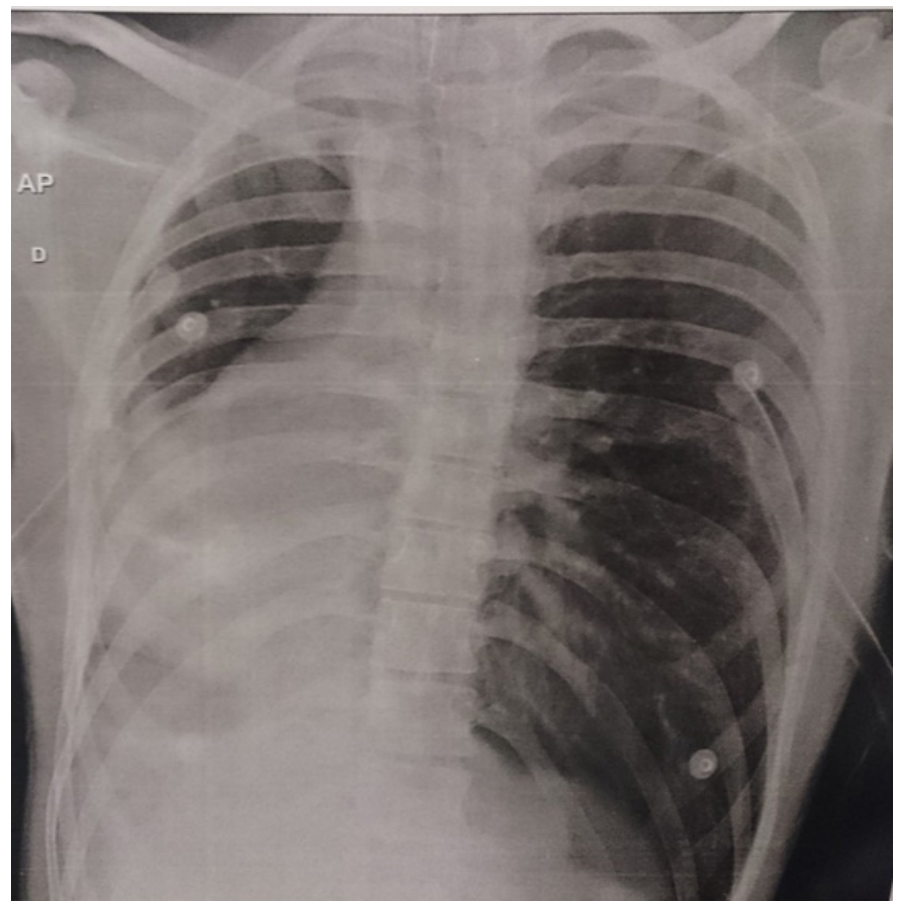

Figura 2. Tomografia computadorizada de tórax evidenciando pulmão esquerdo vicariante e circulação pulmonar normal com desvio das estruturas mediastinais para direita devido à agenesia pulmonar à direita. (Laudo Radiologista)

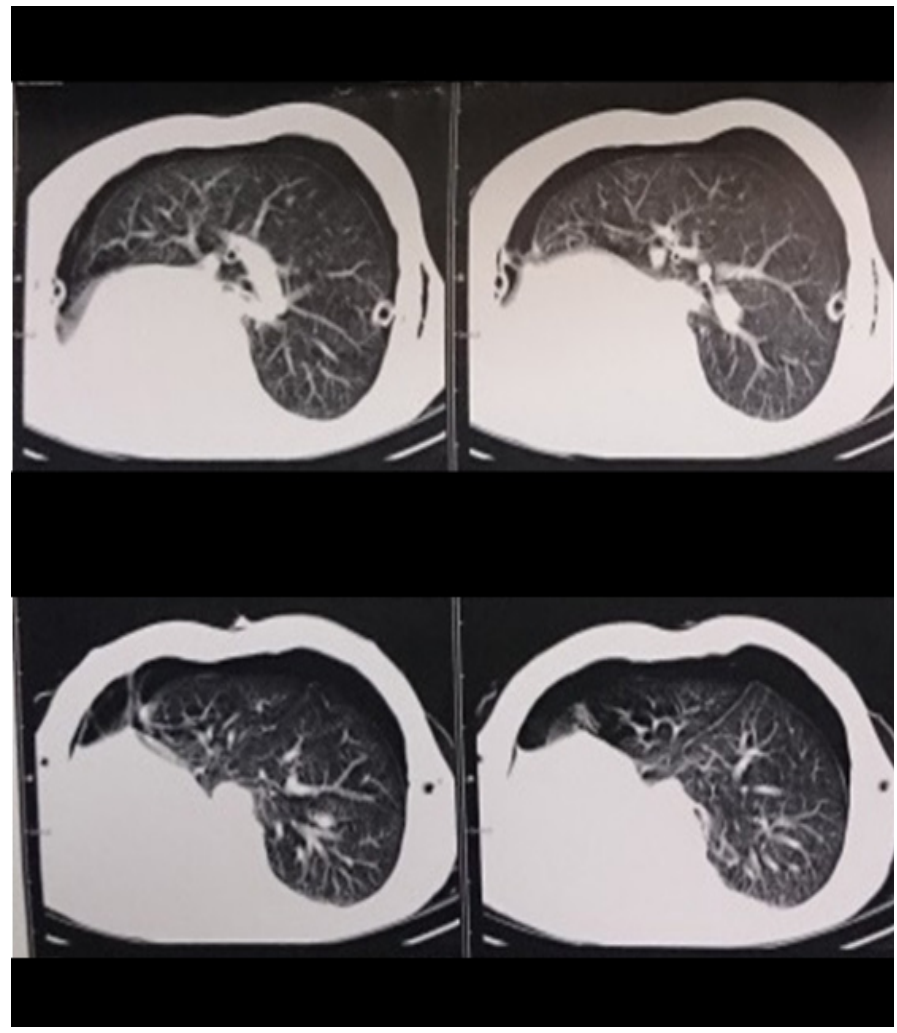


Durante a anamnese, a família relatou que o paciente tem histórico de doença pulmonar desde os 4 meses de vida, sendo acompanhado por pneumologista em Cuiabá / MT. Relatou, ainda, quadro alérgico de repetição quando exposto a poeira, a insetos e a uso de ALENIA; fez uso ainda de PURAN T4 por um ano, porém parou de usar por conta própria há mais ou menos 1 mês. A família negou outras comorbidades e uso de drogas ilícitas.

O diagnóstico médico na UTI foi de Agenesia Pulmonar Direita, Dextrocardia e Pneumotórax Espontâneo Maciço Pós-PCR. Os diagnósticos de enfermagem baseado na NANDA International (2015) foram: risco de débito cardíaco diminuído, padrão respiratório ineficaz, perfusão tissular periférica ineficaz, risco de infecção, troca gasosa ineficaz, ventilação espontânea prejudicada, risco de glicemia instável, risco de lesão.

No terceiro dia de internação na UTI, o paciente estava respondendo ao tratamento, às intervenções de enfermagem, mantinha-se em parâmetros ventilatórios mínimos e enquadrava-se nos demais critérios para desmame. Realizouse a extubação, e houve preservação de um bom padrão respiratório. À avaliação neurológica, obteve-se escala de Coma de Glasgow 15/15, e, no quarto dia de internação, o paciente recebeu alta da UTI para enfermaria de clínica médica sem déficits neurológicos e nenhuma outra complicação clínica oriunda da internação, além das já preexistentes.

O presente artigo é um estudo de caso e obteve a anuência do Comitê de Ética e pesquisa (CEP) da FACIMED, sob Protocolo no 1809223 e CAAE:60435716600005298, conforme recomenda a Resolução 466 de 2012 do Conselho Nacional de Saúde.

\section{DISCUSSÃO}

As malformações congênitas surgem no período embrionário e estão presentes desde o nascimento. Tais alterações podem ser de ordem estrutural, funcional ou metabólica, causando anomalias físicas ou mentais aos indivíduos ${ }^{8}$.

A causa da maioria das malformações congênitas é considerada idiopática; entretanto, os agentes etiológicos mais bem identificados são as anomalias cromossômicas (fatores genéticos), os teratógenos (fatores ambientais) e a herança multifatorial (genética e ambiental) ${ }^{9}$.

Estima-se que cerca de 2 a 3\% dos recém-nascidos são portadores de uma ou mais malformações congênitas, sendo elas responsáveis por $20 \%$ da mortalidade neonatal e 30 a $50 \%$ da mortalidade perinatal em países desenvolvidos. As malformações congênitas representam cerca de 11,2\% dos óbitos infantis no Brasil, sendo a maior causa de mortalidade nessa categoria ${ }^{10}$.

Pacientes com malformações pulmonares congênitas podem manifestar sintomas respiratórios ao nascimento, enquanto outros podem permanecer assintomáticos por longos períodos. Quando diagnosticados tardiamente, esses pacientes apresentam algum tipo de complicação, comumente infecções pulmonares. Dessa forma, torna-se difícil obter dados consistentes e reais sobre a prevalência dessas alterações, mas estima-se que $10 \%$ dos casos são reconhecidos ao nascimento e que outros $14 \%$ serão diagnosticados até os 15 anos de idade ${ }^{1}$. Compreender o diagnóstico é um processo permeado por inquietudes. A família busca as possíveis razões que possam explicar a causa da anomalia, procurando auxílio em diferentes especialidades médicas, incluindo a genética e ainda informações de fontes empíricas e nos saberes populares ${ }^{11}$.

Para um diagnóstico preciso, é essencial o uso de exames de imagem. A radiografia ou tomografia de tórax apresenta uma hiperinsuflação do pulmão remanescente com deslocamento do mediastino para o lado afetado. É fundamental o estudo ecocardiográfico para descartar anomalias cardíacas podendo ser usada, também, a ressonância magnética para pesquisa de malformação vascular e broncoscopia para confirmação diagnóstica $^{5,12}$.

Os pacientes assintomáticos, ao nascer, podem evoluir com tosse, hemoptise e pneumonias de repetição, sendo esta última o achado mais comum, ou ainda permanecerem assintomáticos e terem seu diagnóstico de forma acidental (15,5\% nos casos de sequestrações intralobares e $10 \%$ nos casos de sequestrações extralobares). O quadro dos outros pacientes pode evoluir com complicações, como hemoptise, hemotórax maciço, complicações cardiovasculares, infecções fúngicas ou bacterianas, tumores benignos e tumores com degeneração maligna ${ }^{1}$.

A apresentação clínica é variada. Em alguns casos, os sintomas se manifestam no momento do nascimento como uma síndrome de dificuldade respiratória. Em outros, os pacientes permanecem assintomáticos até a idade adulta, quando o defeito acaba detectado durante um exame de rotina ${ }^{5}$.

A agenesia pulmonar é uma malformação que pode estar presente sozinha ou acompanhada de outras anormalidades. Embora essa associação de anormalidades não esteja bem definida, acredita-se que aconteçam alterações no arco aórtico no período embrionário. As principais malformações são hipoplasia dos músculos, do hemitórax ipsilateral, estenose da traqueia, atresia do esôfago, hérnia diafragmática, microftalmia, anormalidades cardiopulmonares, alterações nos ossos da face, entre outras ${ }^{7}$.

Pacientes que apresentam agenesia pulmonar, mas que permanecem assintomáticos, não necessitam de tratamento. Contudo, quando apresentam alguma outra complicação pulmonar, necessitam de rápida intervenção, sendo a hipertensão pulmonar a mais frequente nesses tipos de pacientes, fazendo-se necessária uma avaliação mais criteriosa, devido ao estreitamento do leito vascular pulmonar que, se associado a uma cardiopatia congênita (shunt esquerdodireito), pode evoluir para uma doença vascular irreversível ${ }^{5}$. 
Uma das ações terapêuticas inclui a criação de um shunt aortopulmonar ou a restauração da continuidade entre artéria pulmonar principal e hilar, quando o diagnóstico for feito em idade precoce. Em adultos, deve ser considerada, em caso de hemoptise, a realização de pneumectomia ou lobectomia quando há risco de vida ou intratáveis infecções pulmonares. Em casos de não haver melhoras no quadro de pneumotórax hipertensivo, a revascularização pulmonar é uma opção a ser considerada para melhora do quadro de hipóxia ${ }^{13}$.

Nesse sentido, deve haver uma avaliação minuciosa dos pacientes a fim de minimizar consequências irremediáveis decorrentes de um diagnóstico tardio.

Sendo assim, a utilização da sistematização da assistência de enfermagem (SAE) é um importante recurso que viabiliza a organização da assistência pelo enfermeiro e pode, simultaneamente, utilizar sistemas de classificação da prática de enfermagem que permitem planejamento e avaliação da assistência que podem detectar indicadores clínicos sugestivos de patologias, como a agenesia pulmonar, gerando, consequentemente, melhora no prognóstico do paciente ${ }^{14}$.

O processo de enfermagem (PE) é composto de cinco fases interligadas e inter-relacionadas. O sistema de classificação é baseado em práticas e evidências cientificas, julgamento clínico e tomada de decisão que o profissional terá como base para implementar suas ações ${ }^{14,15}$.

Os diagnósticos de enfermagem são importantes indicadores da necessidade de cuidados de enfermagem e oferecem base científica e segura para a assistência de enfermagem, uma vez que as intervenções elaboradas são direcionadas às características definidoras e fatores relacionados - elementos constituintes do diagnóstico de enfermagem da NANDAInternational ${ }^{16}$.

As características definidoras são indicadores clínicos que se reúnem ou se agrupam, como manifestações dos diagnósticos de enfermagem. Os fatores relacionados são condições ou circunstâncias que podem causar ou contribuir para o desenvolvimento do diagnóstico ${ }^{17}$.

Portanto, cabe à enfermagem identificar, durante a hospitalização, o diagnóstico do paciente e a intervenção relacionada, visando melhorar seu estado biopsicossocial ${ }^{18}$.

Outrossim, é extremamente relevante que o enfermeiro tenha conhecimento científico sobre a patologia de base do paciente e domine o conhecimento da linguagem padronizada por meio de uma taxonomia validada a fim de possibilitar uma assistência adequada e continuada por parte da equipe de enfermagem, visando a melhores resultados para o paciente.

\section{CONSIDERAÇÕES FINAIS}

A sistematização da assistência de enfermagem é essencial ao exercício da profissão, pois, além de nortear a aplicação de recursos humanos e materiais, otimiza a avaliação da assistência prestada, o que permite verificar o alcance de padrões mínimos de assistência exigidos para a melhora do quadro clínico do paciente, além de apontar fatores e ações que requeiram aprimoramento.

A lei do exercício profissional e o Código de Ética dos profissionais de enfermagem, por meio dos diagnósticos de enfermagem baseados nas evidências, possibilitam a criação de estratégias de intervenções, auxiliando nas condutas clínicas. Nesse sentido, o enfermeiro deve estar apto para estabelecer e gerenciar as ações perante a equipe e o paciente com agenesia pulmonar mediante a realização do diagnóstico e a prescrição de enfermagem, ação considerada privativa do enfermeiro no rol da equipe de enfermagem.

Assim, o enfermeiro exerce um papel fundamental no que diz respeito ao estabelecimento da qualidade da assistência prestada com o intuito da melhora do quadro clínico e da minimização dos riscos de complicações ao paciente.

\section{REFERÊNCIAS}

1. Andrade CF, Ferreira HPDAC, Fischer GB. Malformações pulmonares congênitas. J. bras. pneumol. 2011 Mar-Abr; 37(2): 259-271. doi: http://dx.doi. org/10.1590/S1806-37132011000200017.

2. Ghobadi H, Matin S. Pulmonary Artery Agenesis with Bronchial Asthma. J. Cardiothorac. Med. May 2014; 2(1): 172-175.

3. Lucas M, Graça A, Pereira L, Barreto C, Bandeira T. Malformações pulmonares congénitas - Apresentação de casuística e revisão da literatura. Acta Pediatr Port, v. 33, p. 157-68, 2002. Disponivel em: https://pjp.spp.pt//article/ view/5175/3939.

4. Nandan D, Bhatt GC, Dewan V, Pongener I. Complete right lung agenesis with dextrocardia: an unusual cause of respiratory distress. J Clin Imaging Sci. 2012; $2: 81$.

5. Malcon MC, Cavada MN, Caruso PE, Real LF. Unilateral pulmonary agenesis. J Bras Pneumol. 2012 July-Ago; 38(4): 526-9. doi: http://dx.doi.org/10.1590/ S1806-37132012000400016.
6. Alvarez AJ, Vaccaro MI, Verdejo HP, Villarroel CQ, Puentes RR. Unilateral pulmonary agenesis associated with multiple malformations-a case report [Article in Spanish]. Rev Chil Pediatr.71(1):41-45. 2000. http://dx.doi. org/10.4067/S0370-41062000000100008.

7. Castro MAM Júnior, Castro AP, Rosenberg NP, Zimmermann BS, Müller FM. Agenesia pulmonary. VITTALLE. 2007; 19(2): 73-76.

8. Castro MLS, Cunha CJ, Moreira PB. Frequência das malformações múltiplas em recém-nascidos na cidade de Pelotas, Rio Grande do Sul, Brasil, e fatores sócio-demográficos associados. Cad Saúde Pública. 2006 May; 22(5): 1009-15. doi: http://dx.doi.org/10.1590/S0102-311X2006000500013.

9. Ramos AP. Prevalência de malformações congênitas em recém-nascidos em hospital da rede pública. Rev.Saúde.com. 4(1): 27-42.2008

10. Victora CG, Barros FC. Infant mortality due to perinatal causes in Brazil: trends, regional patterns and possible interventions. São Paulo Méd J. 2001 Jan; 
119(1): 33-42. doi: http://dx.doi.org/10.1590/\$1516-31802001000100009.

11. Bolla BA, Fulconi SN, Baltor MRR, Dupas G. Cuidado da criança com anomalia congênita: a experiência da família. Esc. Anna Nery. 2013; 17(2):284290. Giubergia V. Malformaciones pulmonares congénitas. Neumol Pediatr. 9 (3): 88-94.2014.

12. Giubergia V. Malformaciones pulmonares congénitas. Neumol Pediatr. 2014; 9(3): 88-94.

13. Chaudhry A, Rathore M, Banavaliker J.N. Isolated Right Pulmonary Artery Agenesis with Agenesis of Right Upper Lobe and Bronchiectasis of Right Lower Lobe with Anomalous Arterial Supply from Celiac Axis with Normal Venous Drainage. Indian J Chest Dis Allied Sci. 2014 Jan-Mar; 56(1): 49-52.

14. Pinheiro AM, Tannure MC. SAE: Sistematização da Assistência de
Enfermagem: Guia Prático. 2. ed. Rio de Janeiro: Guanabara Koogan; 2010.

15. Horta WA. Processo de Enfermagem. São Paulo: EPU; 1979.

16. North American Nursing Diagnosis Association. Diagnósticos de Enfermagem da NANDA: definições e classificações 2012-2014. Porto Alegre: Artmed; 2015.

17. Sasso DMTG, Barra DCC, Paese F, Almeida SRW, Rios GC, Marinho MM, et al Processo de enfermagem informatizado: metodologia para a associação da avaliação clínica, diagnósticos, intervenções e resultados. Rev Esc Enferm USP. 2013; 47(1): 242-249. doi: http://dx.doi.org/10.1590/S008062342013000100031.

18. Mariano S, Marques IR. Cardiomiopatia Hipertrófica: atualização e assistência de enfermagem. Rev. bras. enferm. 2007 Set-Out; 60(5): 596-601. doi: http://dx.doi.org/10.1590/S0034-71672007000500021. 